\title{
Synthesis and Characterization of Derivatives Based on 4, - Dimercaptobiphenyl
}

\author{
Shetha F.Al-Zobaydiy* \\ Received 10, April, 2012 \\ Accepted 17, July, 2012
}

\begin{abstract}
:
Starting from 4, - Dimercaptobiphenyl, a variety of phenolic Schiff bases (methylolic, etheric, epoxy) derivatives have been synthesized. All proposed structure were supported by FTIR, ${ }^{1} \mathrm{H}-\mathrm{NMR},{ }^{13} \mathrm{C}-\mathrm{NMR}$ Elemental analysis all analysis were performed in center of consultation in Jordan Universty.
\end{abstract}

Keywords: dimercaptobiphenyl, phenolicschiff bases.

\section{Introduction:}

The development of simple Synthetic routes too widely used organic compound using readily available reagents is one of the main objectives of organic Synthesis. The use of Schiff bases covered a wide area applications, in industry and biological field[1]. In industry (phenolic, methylolic, epoxy) Schiff bases were used as a rubber accelerators[2], atorsantioxidants[3], and corrosion inhibitors[2].They have wide industrial applications as photostabilizers for polyethylene. In analytical chemistry Schiff bases were used for uptake of metal ions[4], and the methylolic resins of some phenolic Schiff bases are used in the accurate determination of trace elements in dilute solution, which is often hindenred due to the sensitivity limits of the equipment used for the purpose[5], in this study number of methylolic, etheric and epoxy Schiff bases[6], were prepared from condensation reaction of aromatic aldehyde with (4,Dimercaptobiphenyl)[7]

\section{Materials And Methods:}

Melting points were determined on Gallenkamp, melting point apparatus and were uncorrected. FTIR spectra of the compounds were recorded on a (SHIMADZU) FTIR. 8300 Spectrometer as KBR-disc,spectra were recorded at 200.13-50.32, respectively using tetra methyl Silane (TMS) as internal standard, (DMSO as solvent. Elemental analysis were run using a perkin-Elmer RE 2400 (C.H.N) analyzer.All analysis were performed in center of consultation/ University of Jordan.All the chemical used were supplied by (Merk, Fluka and BDH) chemicals, the solvents purified by distillation and dried with calcium chloride.

\section{Measurement and Techniques}

The purity of products were investigated by (T.L.C) technique by using a mixture of benzene - ethanol $(5: 5 \mathrm{v} / \mathrm{v})$ as elute and iodine chamber for spot location.

Preparation of [ $4,4^{-}$- [ di-hydrazino biphenyl (1)[7]:

Amixture of $(0.01 \mathrm{~mol}, 2.18 \mathrm{gm})$ [4, Dimercaptobiphenyl ] with $(0.02 \mathrm{~mol}$, $0.64 \mathrm{ml})$ hydrazine hydrate $(99 \%)$ in (10ml) ab. ethanol . Refluxing $6 \mathrm{~h}$ or until ceased $\mathrm{H}_{2} \mathrm{~S}$, then coold the mixture, filtered and crystallized in ethanol.

Synthesis of phenolic Schiff base (2)[6]:

*Department of Chemistry, College of Science for Women, University of Baghdad, Iraq 
Phenolic Schiff base [2] were prepared by well established procedure in the literature[8]condensing $\quad(0.02 \mathrm{~mol}$, $4.9 \mathrm{gm})$ compound (1) with $(0.04 \mathrm{~mol}$, 4.9gm) p-hyroxybenzaldehyde, the purity of the products were investigated by T.L.C.

Synthesis of methylolic phenolic Schiff bases resin (3)[8]

A250ml necks round bottomed flask equipped wih mechanical stirrer, condenser and thermometer, the flask was immersed in water bath, then charged with $(0.05 \mathrm{~mol})$ of phenolic schiff base [2] and $(0.05 \mathrm{~mol}, 1.5 \mathrm{ml})$ formaldehyde solution (41-37)\%. in $50 \mathrm{ml}$ (THF) tetra hydrofuran, the reactants were then mixed gently with ethanolic sodium hydroxide solution $(10 \%)$ was added portion wise to kept the $\mathrm{PH}$ of the reaction mixture (9-10), then the mixture was heated in oil bath $(50-60)^{\circ} \mathrm{C}$ for $3 \mathrm{~h}$, the reaction mixture was cooled $(5-10)^{\circ} \mathrm{C}$ and neutralized with alcoholic phosphoric acid (10\%) solution. Organic layer was separated and purified by dissolving in (THF), then filtered to get rid off salt, the product solution was dried using molecular sieves, the solvent was distilled off by rotoryevapourator, and the final product was dried in vacuum oven for $24 \mathrm{~h}$ at $40^{\circ} \mathrm{C}$.

Synthesis of etheric methylolic Schiff bases (4-5)[9]:

To mixed an appropriate alcohol $(0.08 \mathrm{~mol})$ with $(1 \mathrm{ml})$ concentration () at groats ice $(0-2)^{\circ} \mathrm{C}$, an appropriate methylolic Schiff base [3] (0.4mol) which gently added to the mixture about 1h, Refluxing with increasment of the temperature gradually to the boilding point of the alcohol used,then the mixture was kept at boiling alcohol for $24 \mathrm{~h}$, and neutralized the cooled mixture by using Sodium hydroxide. The resins formed was extracted using chloroform (), dried and evaporated under vacuum, purified by T.L.C. techniques
Synthesis of epoxy phenolic Schiff bases (6-7)[10-11]:

to a mixture of an appropriate $(0.01 \mathrm{~mol})$ phenolic Schiff bases [4-5] with $(0.12 \mathrm{~mol})$ eipychlorohydrine at ice water $(0-5)^{\circ} \mathrm{C}$ for $(15 \mathrm{~min})$, then gently added $(0.14 \mathrm{~mol})$ alcoholic sodium hydroxide in two portions with kept the temperature below $(60-65)^{\circ} \mathrm{C}$. The stirring continued for $2 \mathrm{~h}$, after first addition the aqueous layer was separated from the organic layer, the second portion of solution and stirred further for $1 \mathrm{~h}$, which was for (50min), and then separated.

The organic layer were dried and excess of (eipychlorohydrine) and solvent distilled off under reduced pressure. The formed resins were purified by T.L.C. tachniques

Synthesis schiff bases resins with unstitched ring (8-9)[11]:

A mixture of $(0.01 \mathrm{~mol})$ epoxy compound [7-10] in $(30 \mathrm{ml})(1-2)^{\circ} \mathrm{C}$ Cold methanol with $(0.015 \mathrm{~mol})$ morpholine. Refluxing in oil bath (80$100)^{\circ} \mathrm{C}$ for $(72 \mathrm{~h})$, after cooling the solvent evapourated and purification by T.L.C.

All these synthesis steps were summarized in schemes (1-6), physical properties, FTIR, (), elemental analysis, are listed in tables (1-4), respectively.

\section{Results And Discussion:}

Considerable interest have been expressed in synthesis of schiff bases in recent year due to their industrial and biological importance, starting from 4 , - dihydrazino biphenyl (1):

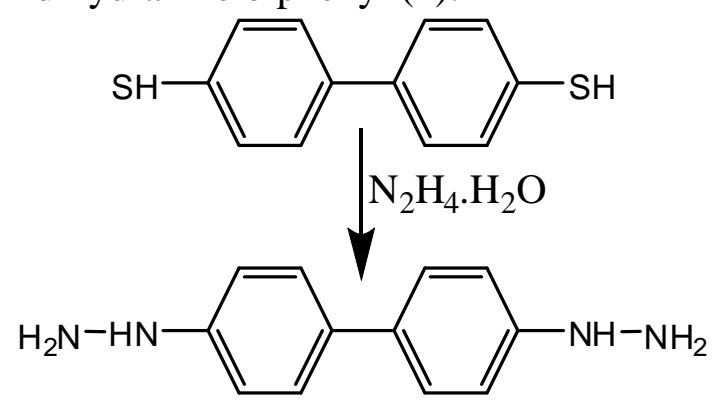




\section{Scheme -1-}

The FTIR spectrum $[12,13]$ showed the strong stretching vibration (3420$3210) \mathrm{cm}^{-1}$ due to $(1361-1385) \mathrm{cm}^{-1}$ for (830) $\mathrm{cm}^{-1}$ for (1,4-disubst), (3080) $\mathrm{cm}^{-1}$ for $(\mathrm{Ar}-\mathrm{H}),(1420) \mathrm{cm}^{-1}$; for $(\mathrm{C}-\mathrm{N}) ;{ }^{1} \mathrm{H}-$ NMR (DMSO-d ${ }_{6}$ ) $\delta:(7.4-7.6) p p m$ due to $(\mathrm{Ar}-\mathrm{H}), \quad(4.1-4.6) \mathrm{ppm}$ due to (4H,NHNH); ${ }^{13} \mathrm{C}-\mathrm{NMR}\left(\mathrm{DMSO}-\mathrm{d}_{6}\right) \delta$ : $(128.2-129.3) \mathrm{ppm}$ due to (aromatic carbons), (9.4 - 9.8)ppm for (Ar-NH).
Elemental analysis (C.H.N) for compound (1) $\mathrm{C}_{12} \mathrm{H}_{14} \mathrm{~N}_{4}$ : [67.29(68.29); $\mathrm{H}, 6.54(7.50) ; \mathrm{N}, 26.17(27.18)]$

There for schiff base (2) prepared through condensation of the corresponding compound (1) with (phydroxy benzaldehyde in refluxing ethanol.

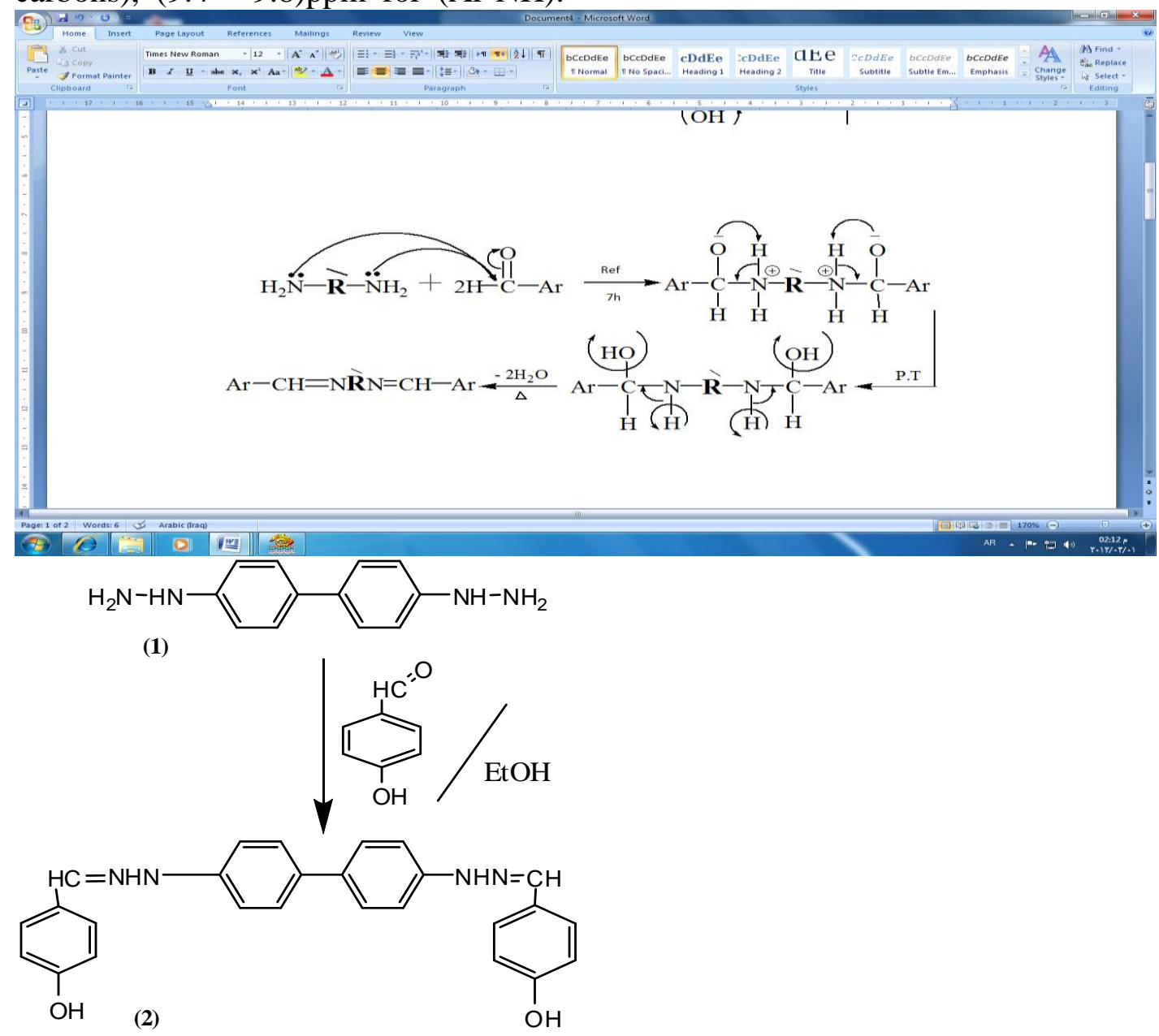

\section{Scheme -2-}

The reaction proceeds by the nucliophilic attach of the nucleophilic nitrogen atom of the amine on the carbonyl group of aldehyde with the loss of water molecular to give a stable compound in good yield, the FTIR spectrum showed the strong bands $(1600) \mathrm{cm}^{-1}$ for $(\mathrm{C}=\mathrm{N})$ combined with diaappearance of stretching bands (3420-3210) $\mathrm{cm}^{-1}$ of $\left(\mathrm{NHNH}_{2}\right)$, strong bands (3400-3240) $\mathrm{cm}^{-1}$ for $(\mathrm{OH})$ and (1150-1160) $\mathrm{cm}^{-1}$ absorption bands for phenolic group (C-O); ${ }^{1} \mathrm{H}-\mathrm{NMR}$ $\left(\right.$ DMSO-d $\left._{6}\right) \delta$ : (7.73-7.85)ppm for (Ar$\mathrm{H}), \quad(10.12-10.16) \mathrm{ppm} \quad(\mathrm{H}, \mathrm{OH}) ;{ }^{13} \mathrm{C}-$ NMR (DMSO-d $\left.\mathrm{d}_{6}\right) \delta$ : (128.2-128.9)ppm (caromatic carbons), (115.2-117.2)ppm for $\quad(\mathrm{C}=\mathrm{CH}), \quad(148.5-152.6) \mathrm{ppm}$ for $(\mathrm{C}=\mathrm{N}),(155.1-156.0) \mathrm{ppm}$ for $(\mathrm{Ar}-\mathrm{OH})$. 
Elemental analysis for compound (2) $\mathrm{C}_{26} \mathrm{H}_{22} \mathrm{~N}_{4} \mathrm{O}_{2}$ : [C,73.93(74.93); $\mathrm{H}$, 5.21(6.20); N, 13.27(14.30)].

Refluxing compound (2) with formaldehyde afforded methylolic resins (3) which is consisting of hydroxyl methylene group $\left(-\mathrm{CH}_{2} \mathrm{OH}\right)$ known as methylol groups which are chemically very reactive functional groups:

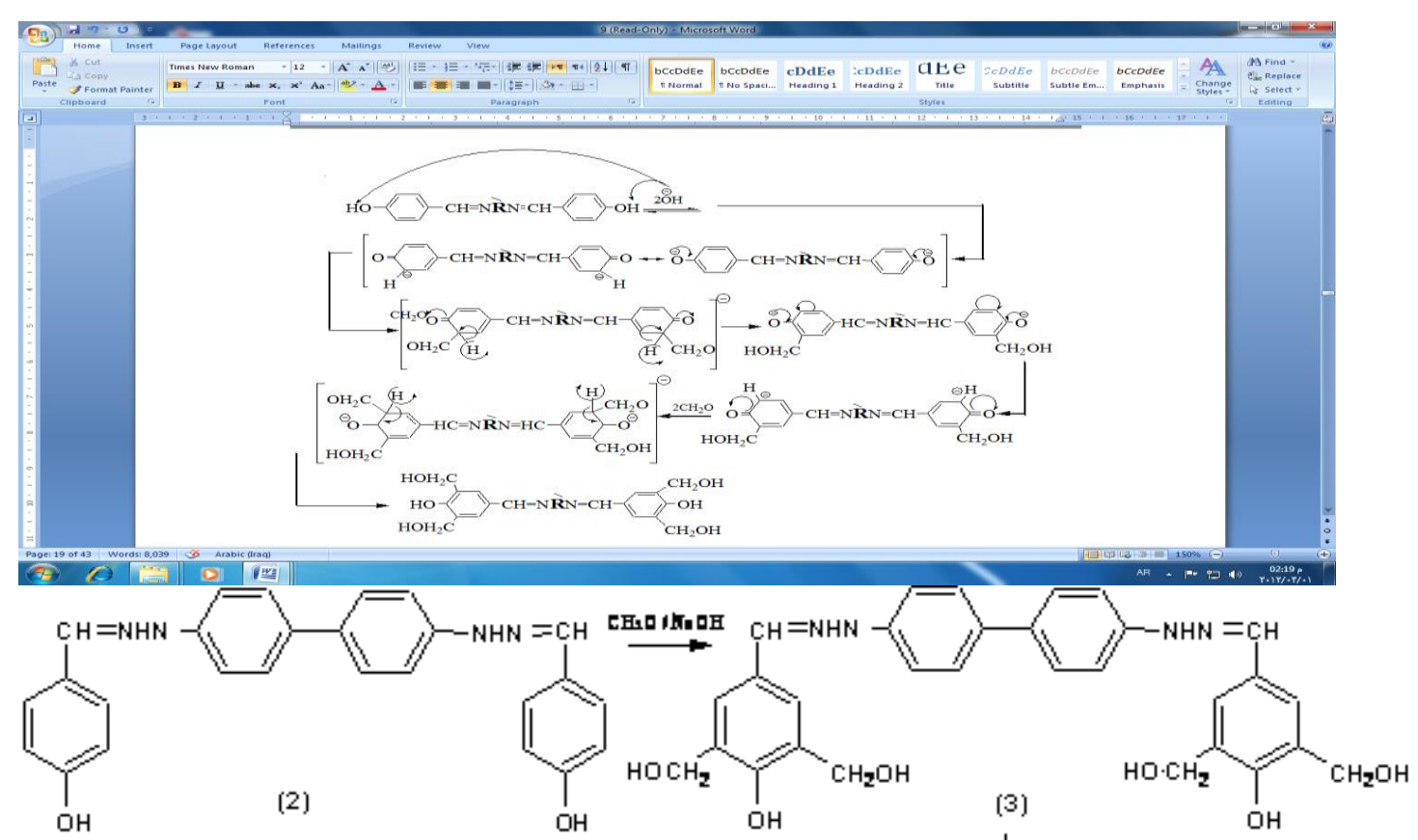

\section{Scheme -3-}

Compound (3) indicated by (FTIR) spectrum of abroad stretching bands at (3465-3292) $\mathrm{cm}^{-1}$ for methylolic groups combined with strong stretching bands at $(2954-2896) \mathrm{cm}^{-1}$ for aliphatic $\left(\mathrm{CH}_{2}\right)$; ${ }^{1} \mathrm{H}-\mathrm{NMR}\left(\mathrm{DMSO}-\mathrm{d}_{6}\right) \quad \delta:(7.7-7.9) \mathrm{ppm}$ due to (Ar-H), (10.3-11.1) ppm for $(\mathrm{H}, \mathrm{OH}),(1.50-2.20) \mathrm{ppm}$ for $\left(\mathrm{t}, 2 \mathrm{H}, \mathrm{CH}_{2}\right)$;
${ }^{13} \mathrm{C}-\mathrm{NMR}(\mathrm{DMSO}) \delta:(126.5-126.8) \mathrm{ppm}$ (aromatic carbons), (115.6-115.8)ppm for $(\mathrm{C},=\mathrm{CH}),(154.8-154.9) \mathrm{ppm}$ for (Ar$\mathrm{OH})$. Elemental analysis for compound (3) $\mathrm{C}_{30} \mathrm{H}_{30} \mathrm{~N}_{4} \mathrm{O}_{6}:[\mathrm{C}, 66.42(67.40) ; \mathrm{H}$, 5.54 (6.50); N, 10.33(11.30)].

Compound (3) similarly react with (methanol, propanol) afforded etheric resins (4-5):

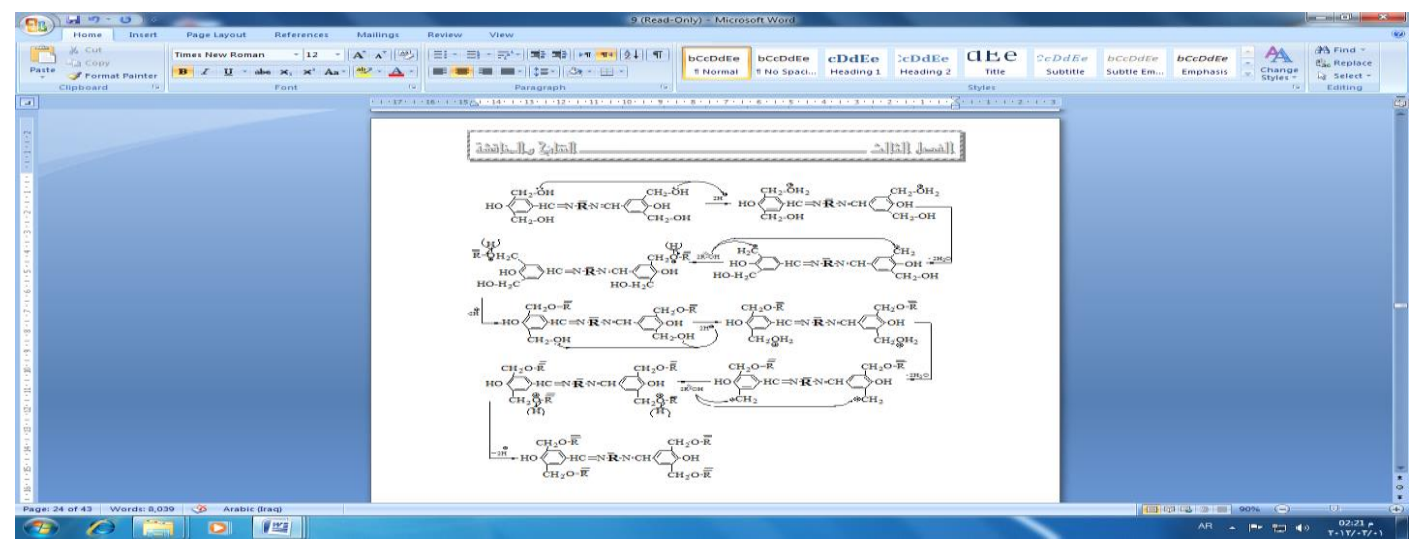




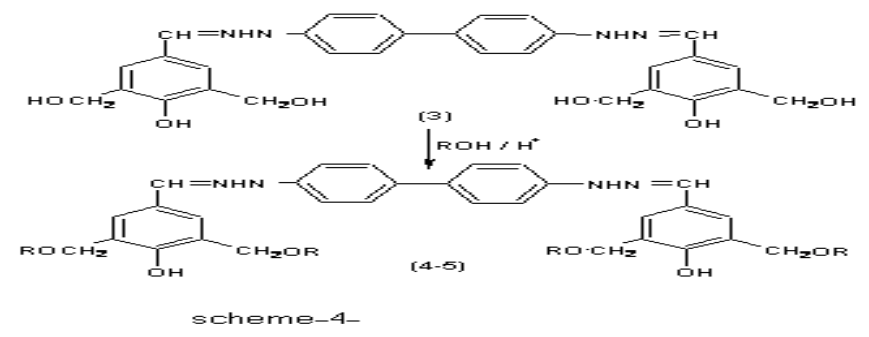

$\mathrm{P}=-\mathrm{CH3} .-\mathrm{C3H7}$

Compound [4] indicated in FTIR spectrum of stretching vibration is more sharp bands at $(2980-2975) \mathrm{cm}^{-1}$ for $\left(\mathrm{CH}_{2}\right)$ and strong vibration at (12261168) $\mathrm{cm}^{-1}$ for (C-O-C); ${ }^{1} \mathrm{H}-\mathrm{NMR}($ DMSO-d $\left.{ }_{6}\right) \delta$ : (7.3-7.6)ppm due to (Ar$\mathrm{H}),(2-2.2) \mathrm{ppm}$ for $\left(3 \mathrm{H}, \mathrm{CH}_{3}\right),(10.01-$ $10.21) \mathrm{ppm}$ for $(\mathrm{H}, \mathrm{OH})$; ${ }^{13} \mathrm{C}$ NMR(DMSO-d 6 ) $\delta: \quad((11.5-116.4) \mathrm{ppm}$ due to (aromatic carbons), (149- 151)ppm due to $(\mathrm{C}=\mathrm{N}), \quad(154.2-$ 154.9)ppm due to (Ar-OH), 972.172.6)ppm for (C-O-C).Elemental analysis for compound [4] $\mathrm{C}_{34} \mathrm{H}_{38} \mathrm{~N}_{4} \mathrm{O}_{6}$ : $[\mathrm{C}, 68.23(69.23) ; \mathrm{H}, 6.35(7.33) ; \mathrm{N}$, 9.37(10.30)].

Similarly react compounds (4-5) with eipychlorohydrine afforded (6-7) derivatives respectively.
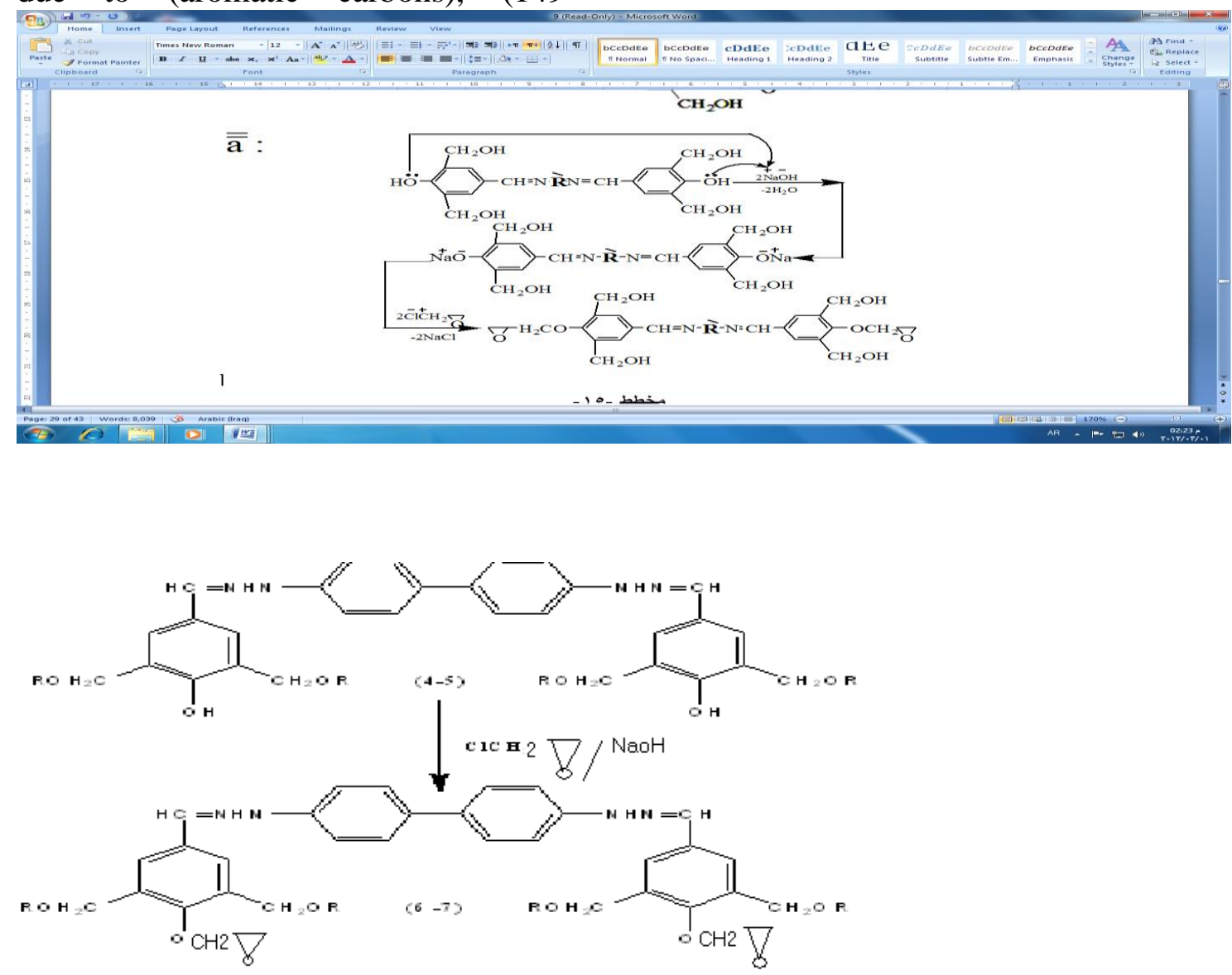

\section{Scheme -5-}

Compound (6) obtained FTIR spectrum for sharp bands for oxarine absorption at (948-995) $\mathrm{cm}^{-1}$ and strong bands (2931-2873) $\mathrm{cm}^{-1}$ for $\left(\mathrm{CH}_{2}\right)$, (11301090) $\mathrm{cm}^{-1}$ for $(\mathrm{C}-\mathrm{O}-\mathrm{C}) ;{ }^{1} \mathrm{H}-$ 
NMR(DMSO-d 6 ) $\delta:(7.2-7.5) \mathrm{ppm}$ for

Elemental analysis for compounds (6) (Ar-H), (1.9-2.1)ppm due to $\left(3 \mathrm{H}, \mathrm{CH}_{3}\right)$; $\mathrm{C}_{38} \mathrm{H}_{42} \mathrm{~N}_{4} \mathrm{O}_{8}:[\mathrm{C}, \quad 66.86(67.80) ; \quad \mathrm{H}$, ${ }^{13} \mathrm{C}-\mathrm{NMR} \quad\left(\mathrm{DMSO}_{\mathrm{d}}\right) \quad \delta$ : (129.36.61(7.16); N, 8.21(9.21)]. Finally (6-7) 130.1)ppm (aromatic carbons), (2.12.3)ppm for $\left(\mathrm{CH}_{2}\right.$, oxarine), (72.6compounds react with morpholine a corresponding (8-9) respectively.

72.9)ppm due to (C-O-C).
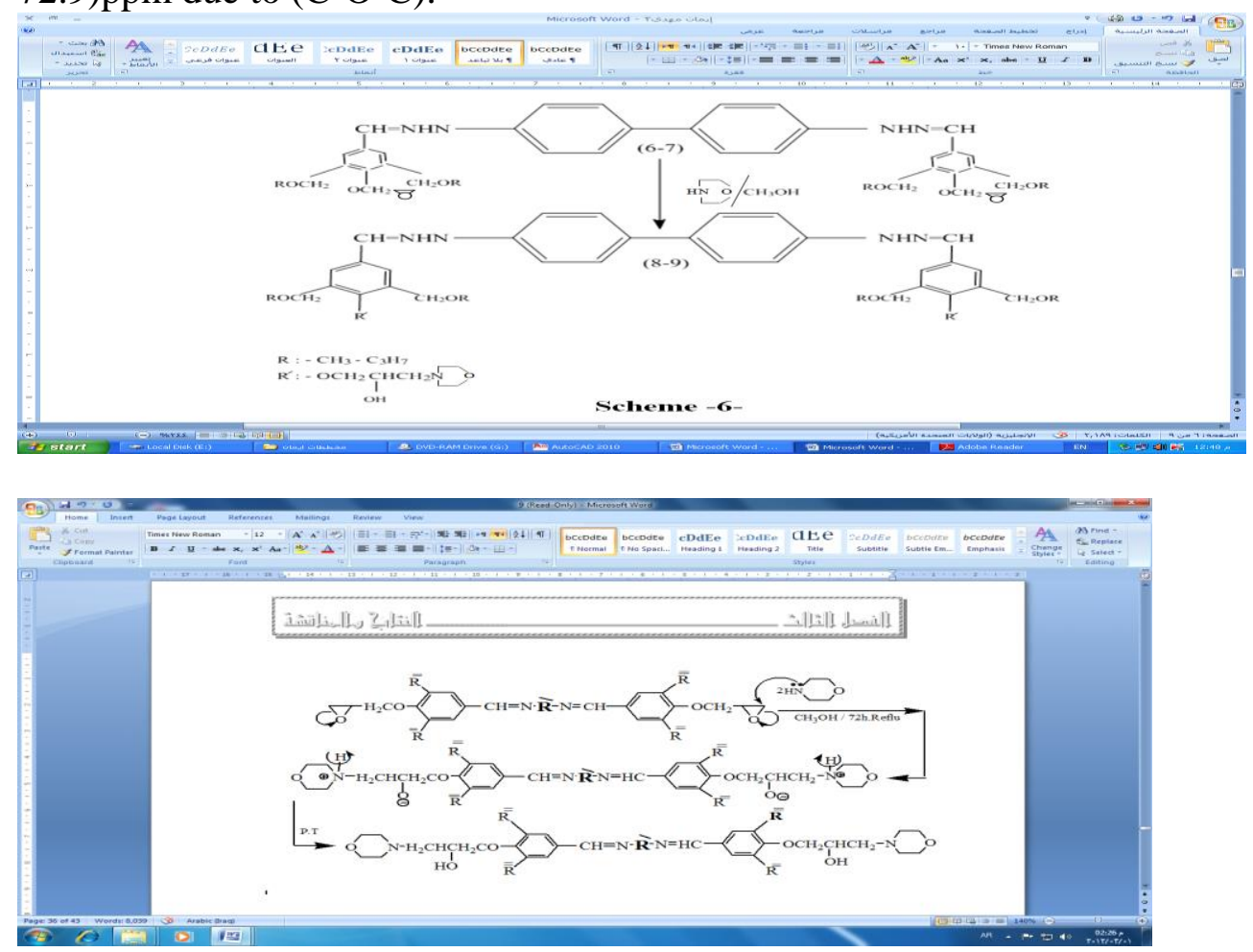

\section{Scheme -6-}

The FTIR spectrum of compound (8) obtained increasing in stretching vibration of $(\mathrm{OH})$ at $(3479-3437) \mathrm{cm}^{-1}$ and (1220-1225) $\mathrm{cm}^{-1}$ for (C-O), (12681238) $\mathrm{cm}^{-1}$ due to $(\mathrm{C}-\mathrm{O}-\mathrm{C}) ;{ }^{1} \mathrm{H}-$ NMR(DMSO- $\left.\mathrm{d}_{6}\right) \quad \delta$ : (7.8-7.9)ppm due to $(\mathrm{Ar}-\mathrm{H}),(9.7-9.8)$ due to $(\mathrm{H}, \mathrm{OH})$, (1.39-1.41)ppm for $\left(2 \mathrm{H}, \mathrm{CH}_{2}\right) ;{ }^{13} \mathrm{C}$ NMR(DMSO-d $\left.{ }_{6}\right) \delta:(72.8-72.9) p p m$ due (C-O-C), (129.3-130.1)ppm for (aromatic carbons). Elemental analysis for compounds [8] $\quad \mathrm{C}_{48} \mathrm{H}_{62} \mathrm{~N}_{6} \mathrm{O}_{10}$ :
$[\mathrm{C}, 65.53(66.52) ; \mathrm{H}, 7.03$ (8.04); N, 9.52(10.50)].

All these steps were summarized in schemes (1-6) physical properties of all mentioned and other details [FTIR, Elemental analysis, ${ }^{1} \mathrm{H}-\mathrm{NMR},{ }^{13} \mathrm{C}$ NMR,] , data are listed in tables (1-5) respectively.

Table 1:Dapcited physical properties for (1-12) compounds

\begin{tabular}{|l|l|l|l|l|l|l|l|l|l|l|l|}
\hline No & $\begin{array}{l}\text { Molecular } \\
\text { formula }\end{array}$ & $\begin{array}{l}\text { M-P } \\
C^{\text {o }}\end{array}$ & Colour & Yield\% & $\begin{array}{l}\text { Purification } \\
\text { solvent }\end{array}$ & $\begin{array}{l}\text { Comp } \\
\text { No }\end{array}$ & $\begin{array}{l}\text { Molecular } \\
\text { formula }\end{array}$ & $\begin{array}{l}\text { M-P } \\
C^{\circ}\end{array}$ & Colour & Yield\% & $\begin{array}{l}\text { Purification } \\
\text { solvent }\end{array}$ \\
\hline 1 & $\mathrm{C}_{12} \mathrm{H}_{14} \mathrm{~N}_{4}$ & $\begin{array}{l}222- \\
224\end{array}$ & Orange & 87 & Ethanol & 6 & $\mathrm{C}_{42} \mathrm{H}_{46} \mathrm{~N}_{4} \mathrm{O}_{8}$ & Oily & Brown & 72 & THF \\
\hline 2 & $\mathrm{C}_{26} \mathrm{H}_{24} \mathrm{~N}_{4} \mathrm{O}_{2}$ & $\begin{array}{l}200- \\
902\end{array}$ & Orange & 75 & THF & 7 & $\mathrm{C}_{48} \mathrm{H}_{62} \mathrm{~N}_{4} \mathrm{O}_{8}$ & Oily & Brown & 75 & THF \\
\hline 3 & $\mathrm{C}_{30} \mathrm{H}_{30} \mathrm{~N}_{4} \mathrm{O}_{6}$ & Oily & Redish & 75 & THF & 8 & $\mathrm{C}_{48} \mathrm{H}_{64} \mathrm{~N}_{4} \mathrm{O}_{10}$ & Oily & Brown & 70 & Methanol \\
\hline 4 & $\mathrm{C}_{34} \mathrm{H}_{38} \mathrm{~N}_{4} \mathrm{O}_{6}$ & Oily & $\begin{array}{l}\text { Pale } \\
\text { brown }\end{array}$ & 70 & THF & 9 & $\mathrm{C}_{54} \mathrm{H}_{80} \mathrm{~N}_{4} \mathrm{O}_{10}$ & Oily & Brown & 70 & Methanol \\
\hline 5 & $\mathrm{C}_{42} \mathrm{H}_{54} \mathrm{~N}_{4} \mathrm{O}_{6}$ & Oily & $\begin{array}{l}\text { Pale } \\
\text { brown }\end{array}$ & 70 & THF & & & & & & \\
\hline
\end{tabular}


Table 2: FTIR spectral data of (1-9) compounds

\begin{tabular}{|c|c|c|c|c|c|c|}
\hline Comp No & $\mathrm{V}_{\mathrm{OH}}$ & $\mathrm{V}_{\mathrm{CH} 2}$ & $\mathrm{~V}_{\mathrm{C}-\mathrm{O}-\mathrm{C}}$ & $\mathrm{V}_{\mathrm{C}=\mathrm{N}}$ & $V_{C=C}$ & Others \\
\hline 1 & - & - & - & - & $\begin{array}{l}1597 \\
1580\end{array}$ & $\begin{array}{l}\mathrm{V}_{\mathrm{NHNH} 2}(3450-3210) \\
\mathrm{V}_{\mathrm{N}-\mathrm{N}}(1535)\end{array}$ \\
\hline 2 & $\begin{array}{l}3400 \\
3300\end{array}$ & - & 1600 & & 1586 & $\begin{array}{l}\mathrm{V}_{\mathrm{C}-\mathrm{O}}(1200) \\
\mathrm{V}_{\mathrm{C}-\mathrm{O}}(1145)\end{array}$ \\
\hline 3 & $\begin{array}{l}3465 \\
3292 \\
\end{array}$ & $\begin{array}{l}2954 \\
2896\end{array}$ & 1612 & & 1575 & $\mathrm{~V}_{\mathrm{C}-\mathrm{O}}(1196)$ \\
\hline 4 & $\begin{array}{l}3400 \\
3255\end{array}$ & $\begin{array}{l}2980 \\
2975\end{array}$ & 1620 & & 1570 & $\mathrm{~V}_{\mathrm{C}-\mathrm{O}}(1190)$ \\
\hline 5 & $\begin{array}{l}3380 \\
3250\end{array}$ & $\begin{array}{l}2960 \\
2889\end{array}$ & 1620 & & 1560 & $\mathrm{~V}_{\mathrm{C}-\mathrm{O}}(1200)$ \\
\hline 6 & $\begin{array}{l}3310 \\
3250 \\
\end{array}$ & $\begin{array}{l}2931 \\
2873 \\
\end{array}$ & 1616 & & 1570 & $(948-995)$ \\
\hline 7 & $\begin{array}{l}3300 \\
3270\end{array}$ & $\begin{array}{l}2960 \\
2940\end{array}$ & 1610 & & 1591 & $(932-985)$ \\
\hline 8 & $\begin{array}{l}3479 \\
3437\end{array}$ & $\begin{array}{l}2970 \\
2975\end{array}$ & 1618 & & 1585 & $\begin{array}{l}\mathrm{V}_{\mathrm{C}-\mathrm{O}}(1220) \\
\mathrm{V}_{\mathrm{C}-\mathrm{O}}(1225)\end{array}$ \\
\hline 9 & $\begin{array}{l}3450 \\
3421 \\
\end{array}$ & $\begin{array}{l}2950 \\
2945 \\
\end{array}$ & 1620 & & 1587 & $\begin{array}{l}\mathrm{V}_{\mathrm{C}-\mathrm{O}}(1218) \\
\mathrm{V}_{\mathrm{C}-\mathrm{O}}(121)\end{array}$ \\
\hline
\end{tabular}

Table $3:{ }^{1} \mathrm{H}-\mathrm{NMR}$ and ${ }^{13} \mathrm{C}-\mathrm{NMR}$ spectral data for some compounds

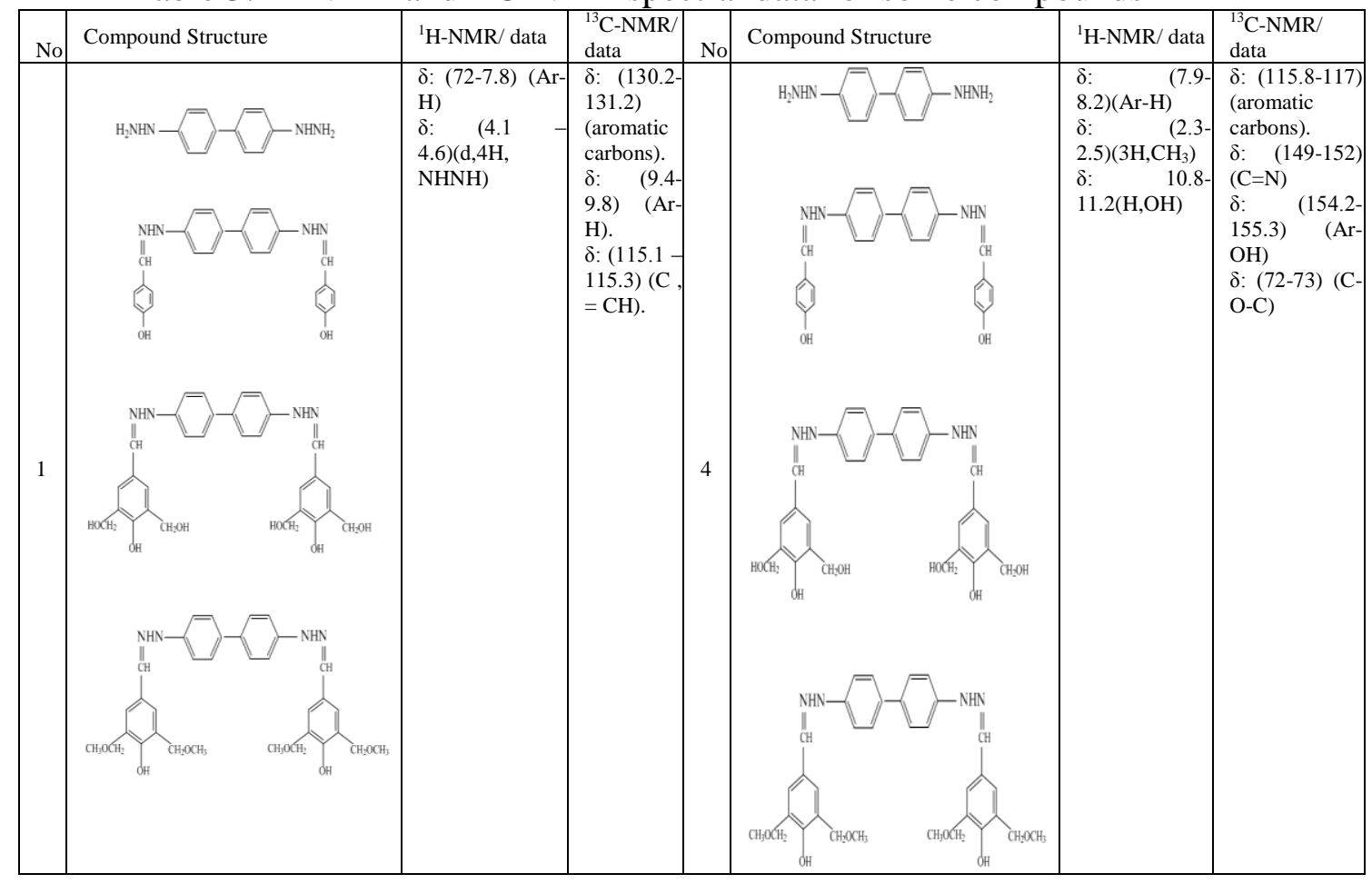




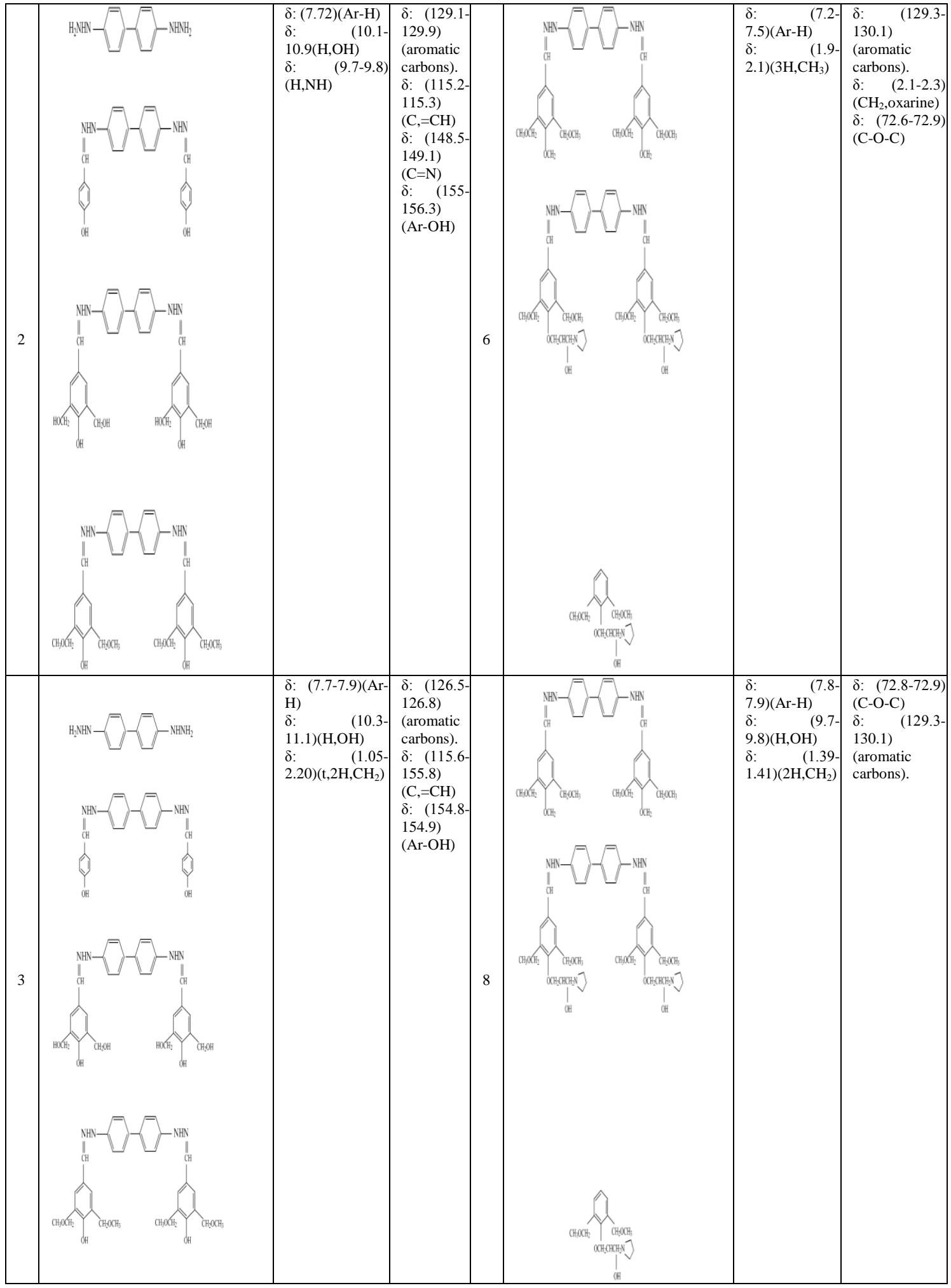


Table 4:Depacited Elemental analysis (C.H.N) for some compounds

\begin{tabular}{|c|c|c|c|c|c|c|c|}
\hline \multirow[t]{2}{*}{ No. } & \multicolumn{3}{|c|}{$\begin{array}{l}\text { (C.H.N) analysis calculated } \\
\text { (found) }\end{array}$} & \multirow{2}{*}{$\begin{array}{l}\text { Comp } \\
\text { No. }\end{array}$} & \multicolumn{3}{|c|}{$\begin{array}{l}\text { (C.H.N) analysis calculated } \\
\text { (found) }\end{array}$} \\
\hline & $\% \mathrm{C}$ & $\% \mathrm{H}$ & $\% \mathrm{~N}$ & & $\% \mathrm{C}$ & $\% \mathrm{H}$ & $\% \mathrm{~N}$ \\
\hline 1 & $\begin{array}{l}67.29 \\
(68.29)\end{array}$ & $\begin{array}{l}6.54 \\
(7.50)\end{array}$ & $\begin{array}{l}26.17 \\
(27.18)\end{array}$ & 4 & $\begin{array}{l}68.23 \\
(68.23)\end{array}$ & $\begin{array}{l}6.35 \\
(7.33)\end{array}$ & $\begin{array}{l}9.37 \\
(10.30)\end{array}$ \\
\hline 2 & $\begin{array}{l}73.93 \\
(74.93)\end{array}$ & $\begin{array}{l}5.21 \\
(6.20)\end{array}$ & $\begin{array}{l}13.27 \\
(14.30)\end{array}$ & 6 & $\begin{array}{l}66.86 \\
(67.80)\end{array}$ & $\begin{array}{l}6.61 \\
(7.16)\end{array}$ & $\begin{array}{l}8.21 \\
(9.21)\end{array}$ \\
\hline 3 & $\begin{array}{l}66.42 \\
(67.40)\end{array}$ & $\begin{array}{l}5.54 \\
(6.20)\end{array}$ & $\begin{array}{l}10.33 \\
(11.33)\end{array}$ & 8 & $\begin{array}{l}65.53 \\
(66.52)\end{array}$ & $\begin{array}{l}7.03 \\
(8.04)\end{array}$ & $\begin{array}{l}9.52 \\
(10.50)\end{array}$ \\
\hline
\end{tabular}

\section{References:}

1. Yang,H.J Sun, W.H. and Zhima.Z.L.2002,"The Rapid Synthesis DF Shiff-Base without Solvent

underMicrowaveIrradiation",Chines e Chemical letters, 13(1): 3-6.

2. Zhao,C.andPizzj,A .Fast advancement and hardening acceleration of low condensation alkaline PFresines by esters and copolymerized urea" 1999,J.Appliedpoly;74: 359-378.

3. Verandas; L.S. and . Fraga C.A.M. , 2005; J. Braz, "Letter in Drug Design \& Discovery"; (2), 62-67.

4. Gupta, V.C and Sutor A. K. 2008, "coordination chemistry Reviews "catalytic Activities of SchiffBase Transition Metal Complexes", 252: 1420-1450.

5. Changi.S.JonesL,Wang.C.j.II,Chu nming.WandRobert.H.C.1998,"Sy nthesis and characterization of new Ruthenium-Based olefin Matathesis catalysts coordinated with Bidentate Schiff-base Ligands"Organo metallic;17(16)3460-3465.

6. Kaya.I, Yildirm.M, Kamacl.M,"Synthesis and characterization of new polyphenols derived from $\mathrm{O}$ diansidines.The effect of substituent on solubility, thermal stability, and electrical conductivity,Optical and electrochemical properties.2009, Eurp.poly.Journal 45,1586-1598.

7. Wadher $\mathrm{S}$.

Puranik,M.P.Karande

.N.A.andYeole .P.G; 2009" Synthesis and Biological evalution of Schiff base of Dapsone and their Derivative As ANTI Microbial Agents "J. Pharm .Tech.1(1) ,22-33.

8. Adam; G.A., 2001, "Chemistry and Technology of Methylolic Resins; "Their Derivative andlPNs. "National Journal of Chemistry, 1: 131-157.

9. Rabek , J. 1980 , " Experimental Methods in Polymer Chemistry", 1: $131-157$.

10. Martin, R.W., 1956 , "The Chemistry of Phenolic Resins"; N.Y., London.

11. Lee; H . and Neville. 1982; 'Hand Book of Epoxy Resins'; Megrow Hill / $2^{\text {nd }}$.

12. Williams;D.H.and Felming,1986; Translated by J.Y, sarkss, "Spectroscopic Methods inOrganic Chemistry", Baghdad University, $1^{\text {st }}$ Edition.

13. Silverstein, R. M. 1981 ,"Specrometric Identification of Organic compound", $4^{\text {th }} \mathrm{Ed}$, part2. 


\section{تحضير وتثخيص بعض مشتقات قواعد شف الفينولية}

*ذى فاضل الزبيلي

| تلية العلوم للبنات /جامعة بغداد

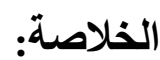

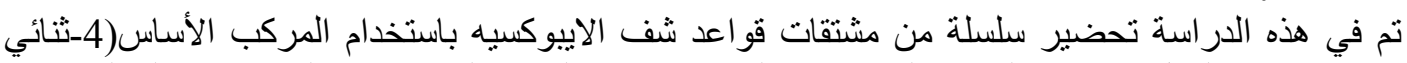

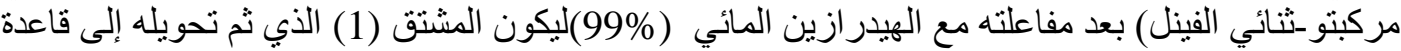

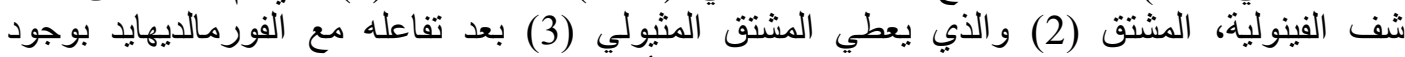

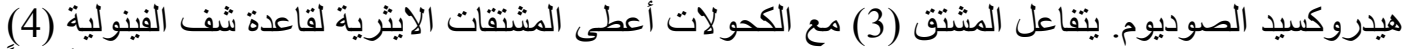

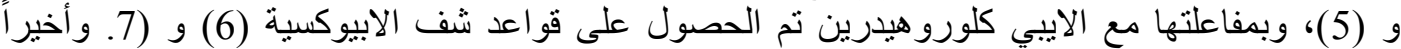

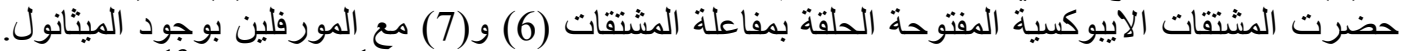

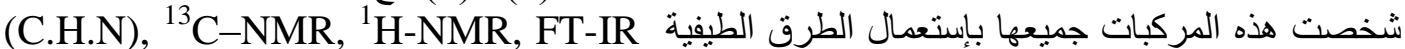
في مركز الاستشار ات في الجامعة الأردنية. 